\title{
EL SUSTRATO ELECTORAL COMUNISTA EN ESPAÑA (1977-2012)
}

\author{
THE ELECTORAL COMMUNIST SUBSTRATUM IN SPAIN (1977-2012)
}

\author{
Alejandro Ruiz-Huerta Carbonell \\ Universidad de Córdoba, España/Spain \\ dp1rucaa@uco.es
}

Recibido/Received: 15/02/2013

Modificado/Modified: 06/06/2013

Aceptado/Accepted: 27/09/2013

\section{RESUMEN}

Se trata de un estudio descriptivo, que analiza la evolución del voto PCE/IU en elecciones generales, municipales, europeas y autonómicas. Se plantean los antecedentes electorales en la historia española, así como la evolución política que llevó al PCE a incorporarse a IU, como alternativa electoral. Se pretende describir el mapa electoral que corresponde con las candidaturas comunistas, que supone la tercera fuerza política del Estado. Se ofrecen datos significativos, en cantidad y porcentaje sobre los municipios españoles en los que más consistencia ha tenido el voto PCE/IU en España. Dichos estudios nos ofrecen la referencia de esa fuerza política española, con amplios apoyos populares en provincias como Córdoba, Sevilla, Valencia, Barcelona, Madrid y Principado de Asturias. Así puede definirse el ámbito público que da su voto a IU.

\section{PALABRAS CLAVE}

Voto de izquierdas, mapa electoral comunista, elecciones, consistencia, ámbito comunista.

\section{SUMARIO}

1. Introducción. 2. El voto comunista en España. 3. Del PCE a IU: un debate inacabable. 4. Los silos del voto PCE/IU 5. La especialidad de las elecciones europeas. 6. Consideración final. Bibliografía

\begin{abstract}
This is a descriptive study about the evolution of the vote to PCE-IU (Spanish Communist Party / United Left) in the four types of election in Spain: generals, locals, autonomics and European. In this work, I study the evolution of the PCE, before and after was created IU on 1984. IU is the third politic party in Spain. There are here significant references, quantitative and in percentage about the Spanish municipalities where PCE/IU is more consistent. This studies offer the reference of this political alternative, with large popular votes in Cordoba, Seville, Valencia, Barcelona, Madrid y Asturias. And this way, we can define the public sphere of PCE-IU in Spain.
\end{abstract}

\section{KEYWORDS}

Left vote, electoral communist map, elections, consistency, communist sphere.

\section{CONTENTS}

1. Introduction. 2. The communist vote in Spain. 3. From PCE to IU: a permanent debate. 4. The silos of communist vote. 5. The specialty of the European elections. 6. Final considerations. References. 


\section{INTRODUCCIÓN}

Es, probablemente Córdoba, el país cordobés como le llamaba Antonio Carbonell, donde existe o ha existido hasta las elecciones de 2012, el mayor silo de voto comunista centrado en el PCE y en IU. Voto comunista que ha supuesto, prácticamente desde 1979, un modelo significativo de gestión en los Ayuntamientos, tras las correspondientes elecciones municipales (EM). Y al estudio de ese voto comunista en España, que tiene a PCE y a IU como destinatarios, se dedica este trabajo, que tiene su base en otro más amplio sobre el voto comunista en Córdoba.

El 15 de Junio de 1977, día en que se celebraron las primeras elecciones democráticas en España, tras 40 años de dictadura, "España fue devuelta a los españoles", en palabras de Julián Marías. Se abría el camino para una segunda oportunidad para la democracia representativa. A partir de aquél día se ponían en marcha los procesos electorales democráticos en España que se mantienen en la múltiple referencia actual de elecciones generales (EG) 11 celebradas, municipales (EM), 9, y autonómicas (EA), 9, además de las europeas (EE) 6, desde nuestra incorporación a sus instituciones en 1986.

Así pues, en estas páginas pretendo definir, a grandes rasgos, cual es el mapa político comunista en España, tras 35 años de elecciones democráticas. Un trabajo descriptivo que afecta a la tercera fuerza política del Estado. Se trata de valorar la importancia de ese voto, mantenido a lo largo del tiempo, a pesar de los múltiples obstáculos que tiene el ejercicio del mismo, como se verá. Un voto que es significativo en Comunidades como Asturias y Cataluña, aun partiendo de la autonomía del antiguo PSUC en Cataluña, pasando por Madrid y Valencia, Córdoba o Sevilla, que son las unidades territoriales de mayor apoyo comunista. Se trata de buscar cual es la base política y territorial del voto comunista en España.

En 1977 parecía que el apoyo al PCE iba a ser muy amplio. No fue así; fue el voto PSOE el más significativo de la izquierda. Pero es importante conocer las referencias del voto comunista (al PCE e IU desde 1986), puesto que se trata de la tercera fuerza política en el Estado. Se esperaba más del voto PCE. Curioso es que un historiador tan conocido como Joseph Fontana hable precisamente de este tema, refiriéndose a "uno de los misterios de la transición", en relación con la importancia del PCE antes del 15 de Junio y después, tras el escaso apoyo electoral que recibe. Una referencia, la del misterio, que se repite en muchos comentarios sobre el tema, aunque creo que las realidades políticas pueden hablar por sí solas.

Se han utilizado para los datos electorales tanto las páginas Web del Ministerio del Interior (MIR), del Congreso de los Diputados y el Senado, los datos digitales que ofrecen los diferentes Parlamentos Autonómicos españoles y, en muchos casos, los mismos datos municipales, así como los datos que presenta el Centro de Análisis y Documentación Política y Electoral de Andalucía.

\subsection{Los antecedentes}

Los inicios del ejercicio del voto en la sociedad occidental, están vinculados al derecho de propiedad; un vínculo entre propiedad y derecho de sufragio, que parece evidente. Por eso la primera manifestación del voto, que se da en los primeros tiempos de los procesos revolucionarios franceses del siglo XVIII, es la idea del sufragio censatario, que limitaba el derecho de voto o sufragio para aquéllas personas que pagaban impuestos al Estado y, por eso, aparecían en un censo, de ahí su nombre.

La primera ley electoral, siguiendo en esta materia la obra de Alejandre (1990), es una Instrucción de Enero de 1810 que preveía, que más de 200 Diputados, de un total de 208, uno 
por cada 50.000 habitantes, fuera elegido por un sistema que se califica enfáticamente de "sufragio universal aunque indirectamente". Posteriormente, la Constitución de 1812 incluye la referencia al sufragio censatario, en cuanto los electores, que eran elegidos por Juntas de Parroquia y de Partido, tenían que alcanzar un determinado nivel de rentas y ser mayores de 25 años.

\subsection{Hacia el sufragio universal}

Un Decreto del Gobierno provisional de 1868 proclamó el sufragio universal como fundamento de las libertades individuales. En 1878 se vuelve al sufragio restringido; en 1890 se vuelve a ampliar, un sufragio universal aun con limitaciones y que el protagonismo terrateniente parece que lo desvirtúa. A este respecto es conocida la opinión de Tuñón de Lara (1971), en su obra sobre el siglo XIX español, de que ese sufragio universal no ha funcionado más allá de las grandes ciudades. Evidentemente, en el mundo rural la realidad era otra, con el peso que mantenía el caciquismo.

Así evoluciona el sufragio hasta la llegada de la Segunda República española, que instaurará el sufragio universal, también para las mujeres. Aunque el sufragio censatario se desmorona paralelamente al auge del universal, a partir de 1877, tras los intentos de una República federal y la nueva Restauración monárquica, el universal parece que se va a imponer, aunque siempre con muchas limitaciones. Y probablemente una de las dificultades que se manifiestan en su consolidación es que, al menos en un primer momento, también se vincula al derecho de propiedad, como el censatario, lo que limitará su ejercicio.

Por tanto, va a ser en la Constitución republicana de la Segunda República, cuando se instaure en España el sufragio universal al establecer en su artículo 52 el sufragio universal igual directo y secreto, para elegir a los Diputados, sin discriminación por motivos de sexo, según el art. 53. Incluso el sufragio universal se extiende a la elección de los Alcaldes, que lo serán "por el pueblo o el Ayuntamiento", artículo 9, y en la elección del Presidente de la República que es elegido, parcialmente, a través de un colegio electoral, elegido por sufragio universal (artículo 68).

\subsection{La aparición electoral del PCE}

El PCE nace en 1920, a través de la Federación de Juventudes socialistas; fue, por tanto, consecuencia de una brecha abierta entre los socialistas españoles, en relación con la organización internacional de la izquierda. Son de Córdoba 6 de los 9 concejales PCE elegidos en Andalucía en las Municipales de 1931. Las primeras elecciones generales, además, en las que el PCE logra algún escaño, son las de diciembre de 1933, en las que Cayetano Bolívar es elegido diputado por Málaga. Salió de la cárcel para ocupar su escaño.

La debilidad del partido, tras años de clandestinidad y represión era grande a pesar de que en 1936 creció ampliamente el número de sus afiliados. De igual forma es un hecho el amplio apoyo a esas candidaturas comunistas que se dan en pueblos de Córdoba, que han mantenido ese voto hasta hoy, así como que en las elecciones de 1933 Córdoba tiene el mayor número de votos PCE, alrededor de 27.000, según han estudiado Trías Bejarano o Moreno Gómez.

\subsection{Algunos datos de participación}

Se incluyen a continuación los porcentajes de abstención/participación en elecciones generales (EG) y municipales (EM); es significativo el nivel de abstención en las EM, superado posteriormente por las EE. 
CUADRO 1: Participación electoral EG y EM

\begin{tabular}{|l|c|l|}
\hline 1. EG & España & Andalucía \\
\hline 1977 & 78.8 & 78.5 \\
\hline 1979 & 68 & 68.6 \\
\hline 1982 & 80 & 78.7 \\
\hline 1986 & 70.5 & 70.8 \\
\hline 1989 & 69.7 & 69.3 \\
\hline 1993 & 76.4 & 76.2 \\
\hline 1996 & 77.4 & 78 \\
\hline 2000 & 68.7 & 68.8 \\
\hline 2004 & 75.7 & 74.8 \\
\hline 2008 & 73.8 & 72.8 \\
\hline 2012 & 71.7 & 70.7 \\
\hline
\end{tabular}

\begin{tabular}{|l|c|l|}
\hline 2. EM 1979 & Sin datos & Sin datos \\
\hline 1983 & - & 66.3 \\
\hline 1987 & 69.4 & 66.7 \\
\hline 1991 & 62.8 & 61.2 \\
\hline 1995 & 69.9 & 68.8 \\
\hline 1999 & 64 & 64.6 \\
\hline 2003 & 67.7 & 65.6 \\
\hline 2007 & 63.3 & 61.6 \\
\hline 2011 & 66.2 & 65.8 \\
\hline
\end{tabular}

Fuente: Datos electorales del MIR.

CUADRO 2: Media global en \% EG y EM.

\begin{tabular}{|l|c|c|}
\hline 3. Media EG & España & Andalucía \\
\hline Participación & 73.9 & 73.6 \\
\hline Abstención & 26.1 & 26.4 \\
\hline 4. Media EM & & \\
\hline Participación & 66.1 & 65 \\
\hline Abstención & 33.9 & 35 \\
\hline
\end{tabular}

Fuente: Elaboración propia sobre datos del Ministerio del Interior (MIR)

\section{EL VOTO COMUNISTA EN ESPAÑA}

Si el PCE ocupó la vanguardia de la lucha antifranquista durante muchos años, en la democracia van a cambiar las tornas y ocupará como PCE o como IU la retaguardia de la democracia parlamentaria española. Algo así como una venganza de la historia aunque tenga distintos protagonistas. Es significativo el contraste con el papel que jugó el PSOE, en la transición y posteriormente, que de no estar en la transición, se convertirá, a partir de 1977 , en la vanguardia de la contienda electoral desde planteamientos inicialmente considerados de izquierda europea.

En un documento del Observatorio electoral de la Federación de Investigaciones Marxistas (Aja, 2006), sobre 18 países con voto de izquierda comunista, su media de apoyo electoral entre 2001 y 2005 se sitúa en torno a un $8 \%$. Un porcentaje visual que define como tercera fuerza política en general, a los distintos partidos y grupos que forman el partido de la Izquierda Europea o el Grupo de la Izquierda Unida Europea. Un dato que avala más el peso 
comunista en Madrid, Barcelona, Asturias o Córdoba, que tiene porcentajes de apoyo a $\mathrm{PCE} / \mathrm{IU}$ en torno al $25 \%$, superando en muchos ámbitos y distintas convocatorias electorales el 35 o el $40 \%$.

Por tanto, la referencia espacial alude a que el voto comunista en España, en concreto sus dos terceras partes, se producen en torno al triángulo que forman las Comunidades de Cataluña, Madrid y Andalucía, que aportan el conjunto electoral más significativo de todo el Estado. Así puede decirse, en el IV Informe FOESSA (1981), que esas tres zonas con más del $45 \%$ de los votantes, contribuyen con el $62.7 \%$ del electorado comunista; a esa realidad solamente habría que añadir el peso del voto comunista en Asturias, lo que es tradicional en la historia electoral, y el de la Comunidad Valenciana, que mantiene también niveles medios de apoyo PCE/IU en porcentajes bastante altos.

\subsection{El debate sobre el comunismo}

El PCE, "el partido", como se le reconocía en la larga noche del franquismo y años después, siempre fue la referencia precisa para hablar del esfuerzo colectivo por la libertad y la democracia que protagonizaron amplios sectores y grupos sociales y políticos, tanto a lo largo del franquismo, como en la transición. Pero a la vez, hay que reconocer igualmente, el enorme peso que el PSOE también tuvo y, especialmente, en los inicios de la aplicación del sistema democrático en España. El predominio PSOE fue tan grande en el conjunto del país que en los años 80 llegó a disponer del 58\% de los escaños en el Congreso de los Diputados, la mayoría de 13 de los 17 Parlamentos autónomos y casi las tres cuartas partes del total de concejales de las localidades de más de 20.000 habitantes. Un predominio electoral muy importante que convierte al PSOE en el partido clave en la construcción del Estado constitucional, a partir de sus éxitos electorales, a la vez que UCD, que hizo lo mismo en la transición, desaparecía de la escena política.

La crisis del movimiento comunista mundial estuvo, también, permanentemente presente en el desarrollo de la democracia en España desde 1977 y afectó muy ampliamente al PCE. Las oscilaciones en sus apoyos populares hay que ponerlas en relación con esa crisis porque va a afectar a su evolución. El primer planteamiento en torno al eurocomunismo parecía impecable, aunque difícilmente fue hecho realidad en una sociedad cada vez más globalizada, y más autónoma; un eurocomunismo que no fue sino estrategia de encuentro europeo de los principales partidos comunistas, excepto el portugués, especialmente vinculado aún a la URSS.

Y, entre la reforma y la ruptura, polémica que aún colea entre los comunistas españoles, entre los errores propios y las tergiversaciones colectivas, además de la enorme influencia del llamado "centralismo democrático", el propio PCE podría llegar al final de un proceso irremediable de desaparición, que se encargó de paliar con su recomposición, en la nueva estrategia de crear Izquierda Unida.

\subsection{La influencia de un sistema electoral desproporcionado}

Valorar el tercer lugar que ocupa el voto comunista en España, no puede hacerse sin tener en cuenta el carácter determinante del sistema electoral; porque dicho sistema, que proviene de 1977, provoca la realidad del bipartidismo y la marginación obligada de los demás partidos presentes en la vida política. De hecho la influencia del sistema o fórmula electoral para trasformar votos en escaños, condiciona y minoriza el voto de varios partidos, entre ellos del PCE/IU, que acaba teniendo una representación parlamentaria mucho menos que proporcional, como es conocido. 
Como es conocido en diversos estudios de la transición, el sistema electoral se hizo realmente para favorecer a los dos partidos más apoyados por el electorado, puesto que parecía que nadie iba a conseguir la mayoría absoluta, aunque lo que aparentaba era la estabilidad del sistema en su momento inicial y, además perseguía explícitamente la reducción de su fragmentación.

El sistema electoral español, creado, por tanto, inicialmente en la transición, favorece a los partidos mayoritarios, presupone la imposibilidad de que el voto sea igual en España y favorece además a los partidos de la derecha, y ello es perfectamente conocido por los expertos y forma parte de una dinámica aun no asumida por el Bloque dominante en España, que forman PP y PSOE, para la necesaria e imprescindible reforma electoral. En un sistema puramente proporcional IU tendría el doble de representación que tiene con el sistema actual. Subiría un 100\% su representación. En 2004 necesitó casi 250.000 votos por escaño. Es lo que suele denominarse la cuota electoral. El número medio que necesita el PP para conseguir un escaño, es de 65.000 votos, cerca de 60.000 el PSOE y prácticamente el doble el PCE/IU, 120.000 votos, que con el paso del tiempo se ha aumentado hasta 200 o 300.000 votos. Esta referencia ha obligado a decir que el sistema electoral español es el menos proporcional de todos los sistemas con fórmulas parecidas.

Pero hay otro factor que condiciona la formación de las Cámaras y su extraña proporcionalidad en relación con los votos. Es la fuerte presencia de diversos nacionalismos existentes en España que en algunos casos, con menos votos que IU, ha sobredeterminado los resultados electorales, los modifica por el protagonismo de opciones nacionalistas en las Cortes Generales y complica aún más el sistema electoral. Existen partidos de ámbito puramente territorial que son beneficiados por el sistema electoral, en contra de algunos partidos que presentan candidaturas en todo el ámbito estatal, que saldrían perjudicados. Es el caso de PCE/IU: siempre consigue ser la tercera fuerza política en España, pero con un número de escaños mucho menor que los que consiguen otras fuerzas, aun cuando el PCE les supera en número de votos.

\subsection{La evolución del voto}

Por último, en el desenvolvimiento del voto pueden destacarse los siguientes datos clave, y tiempos:

$1^{\circ}$ Entre 1977 y 1982, año en que, el triunfo electoral socialista cambia el panorama electoral y político en España, se puede entender que es la etapa de despegue electoral en la democracia española. En ese sentido se produjeron muchos cambios en el sistema de partidos, con la práctica desaparición de la UCD que había protagonizado la gestión de los años más significativos de la transición, entre 1976 y 1979.

$2^{\circ}$ España, entre 1977 y 1990 tenía el mayor índice de abstención de los 12 países europeos, en cinco elecciones generales, un $26.7 \%$. Índice algo superior en las Locales, del $34 \%$. Andalucía mantiene unos niveles algo inferiores al nivel estatal.

$3^{\text {o }}$ 1982-1996 Gestión política del PSOE, que vence en cuatro elecciones generales consecutivas, con una clara tendencia a la baja en el apoyo popular. En esta fase hay que destacar la elaboración y aprobación parlamentaria de la Ley Orgánica de Régimen Electoral General (LOREG) en 1986, que desde entonces rige los procesos generales españoles. La LOREG no modifica apenas la norma vigente hasta entonces, el Decreto Ley de 1977, antes citado, lo que tiene como consecuencia la consolidación de un sistema electoral bipartidista.

$4^{\circ}$ Es significativo el aumento del voto PCE/IU, a medida que hablemos de más población. De hecho es en las ciudades superiores a 50.000 habitantes donde hay mayor porcentaje de voto PCE, incluso superior al 10\%. Alguna excepción se produce en Córdoba provincia. 
5 1996-2004 Desarrollo de la gestión popular (del PP) en el gobierno de España. En 1996 la participación subió, pero no así en 2000, en que el PP consiguió la mayoría absoluta.

$6^{\circ}$ 2004-2011 Nueva gestión socialista. La amplia tasa de volatilidad electoral afecta al voto comunista. Dicha tasa supera a la media europea de la posguerra.

\section{DEL PCE A IU: UN DEBATE INACABABLE}

Cuando en 1986, tras un significativo retroceso electoral del PCE, surge la iniciativa de crear Izquierda Unida, parecía en principio responder a la idea clave de renovar los planteamientos políticos del mismo PCE, abriendo el partido a la sociedad, tratando de llegar a la izquierda real. La coalición se va a crear en el impulso de las movilizaciones para exigir la salida de España de la OTAN; un referéndum convocado confusamente por el Gobierno del PSOE en 1986, mantuvo a España en dicha organización. La respuesta fue amplia desde la izquierda no socialista y desde muchos militantes del propio PSOE, que mantuvieron una gran perplejidad silenciosa frente a la confusión del mismo partido.

En IU siempre, desde su misma fundación, hubo dos posicionamientos, uno el de mantener plena independencia del PSOE y, dos, los partidarios de la idea de casa común de la izquierda. Así, en 1984 se crea una alternativa de izquierdas como posible coalición electoral que pudiera hacer resaltar el peso del PCE, pero abriendo espacios para otras izquierdas. Parece que existen dos perspectivas diferentes del origen de IU. Una sería la propuesta que nace en el PC de Andalucía y lidera Julio Anguita, de crear "Convocatoria por Andalucía" como "un amplio bloque social que pueda sostener una política de progreso". Otra sería la que, propuesta a través de antiguos militantes del PSP en Asturias, lideraría el mismo Secretario General del PCE Gerardo Iglesias.

Los partidos que fundan IU fueron PCE, Partido de Acción Socialista, Izquierda Republicana, PCPE, Federación Progresista, Partido Carlista y Partido Humanista; primero como coalición para ser posteriormente considerada como Movimiento político. Los porcentajes de apoyo PCE/IU, en todo caso, no dejan lugar a dudas de las continuas crisis de la actual coalición, antes y después de su creación en 1986, como se expone en el siguiente cuadro. Crisis que se manifiesta principalmente, en una bajada progresiva y constante de apoyos electorales.

CUADRO 3: Porcentajes de apoyo PCE/IU 1977-2008 EG y EM

\begin{tabular}{|l|l|l|c|}
\hline \multicolumn{2}{|l|}{ Elecciones Generales } & \multicolumn{2}{|l|}{ Elecciones Municipales } \\
\hline Voto PCE 1977-1986 & $8.1 \%$ & Voto PCE 1979-1986 & $10 \%$ \\
\hline Voto IU 1986-1995 & $7.7 \%$ & Voto IU 1986-1995 & $9.3 \%$ \\
\hline Voto IU 1996-2008 & $7.3 \%$ & Voto IU 1996-2008 & $6 \%$ \\
\hline Voto IU 2011 & $6.9 \%$ & Voto IU 2012 & $6.3 \%$ \\
\hline
\end{tabular}

Fuente: MIR

\subsection{Las elecciones municipales}

Cualquier referencia al factor territorial desde la perspectiva electoral, define la zona de influencia del voto PCE-IU. Así en las 13 Comunidades que llamábamos de segundo grado, donde apenas existe la influencia de partidos o alternativas de carácter nacionalista o similar, es donde hay más influencia del voto comunista, aunque sea reducida. Probablemente, además de eso, el dato inicial que merece la pena destacar es la desigual estructura de los 
municipios españoles, que va a condicionar cualquier planteamiento electoral, en las generales, en las municipales o en las autonómicas.

CUADRO 4: El voto PCE/IU en las Generales

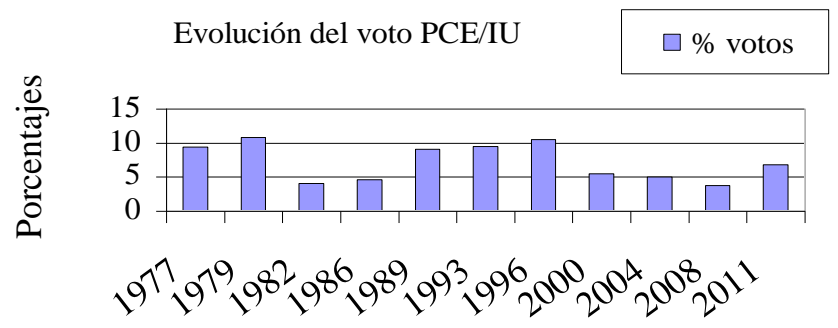

Fuente: Datos electorales del MIR

Efectivamente, más del $90 \%$ de los municipios españoles pueden considerarse rurales, y en ellos vive solamente alrededor del $25 \%$ de la población, a pesar de que en ellos se elige en torno al $80 \%$ de los concejales. En cambio, casi en el 10\% restante de los municipios españoles, que son urbanos, vive más del $75 \%$ de los habitantes y eligen solo el $20 \%$ de los concejales.

CUADRO 5: Porcentajes de apoyo PCE/IU en las EG 1977-2011 S / total votos:

\begin{tabular}{|c|c|c|l|}
\hline EG & $\mathrm{N}^{\text {o de votos }}$ & Porcentaje & \multicolumn{1}{|c|}{ Resultado } \\
\hline 1977 & 1.709 .890 & 9.4 & Crece \\
\hline 1979 & 1.938 .487 & 10.8 & Pierde 50\% electores \\
\hline 1982 & 846.515 & 4.1 & Votos IU \\
\hline 1986 & 935.504 & 4.6 & Crece \\
\hline 1989 & 1.858 .588 & 9.1 & Crece \\
\hline 1993 & 2.253 .722 & 9.5 & Techo electoral \\
\hline 1996 & 2.639 .774 & 10.5 & $\begin{array}{l}\text { Pacto PSOE *(NI). Pierde un } \\
\text { millón de votos }\end{array}$ \\
\hline 2000 & 1.263 .043 & 5.5 & Tendencia a la baja \\
\hline 2004 & 1.284 .081 & 5 & Pierde casi 50.000 votos \\
\hline 2008 & 969.946 & 3.8 & $\begin{array}{l}\text { Amplia subida. Más de } \\
600.000 \text { votos }\end{array}$ \\
\hline 2011 & 1.685 .991 & 6.9 & \\
\hline Media & & 7,2 & \\
\hline
\end{tabular}

Fuente. Elaboración propia sobre datos CADPEA y MIR. *NI: Nueva izquierda.

En la convocatoria de 1979 el PCE presenta candidaturas en 1525 ayuntamientos, para la elección de casi 70.000 concejales y más de 8.000 alcaldes. El PCE conseguirá un total de 3.753 concejales y 236 alcaldes tras la firma de los Acuerdos, citados a continuación, entre el propio PCE y el PSOE.

\subsection{El acuerdo PCE/PSOE de 1979}

Fue un Acuerdo especialmente importante, el que firmaron en 1979 PCE-PSOE, que otorgó la alcaldía en muchos Ayuntamientos, al partido de los dos que hubiera conseguido mayor número de votos. El Acuerdo tenía como contenidos esenciales la democratización 
plena de los Ayuntamientos, la aprobación de Planes de actuaciones urgentes, asegurar eficacia, transparencia y honradez y constituir mayorías estables. El Acuerdo se firma el 18 de Abril de 1979 y es consecuencia de la política de concentración democrática de cara a las primeras municipales, como propuesta de gobierno local. Los resultados del acuerdo son que más de 1500 municipios que representan el $60 \%$ de la población tendrán mayoría de izquierda.

CUADRO 6: los apoyos electorales en las EM

\begin{tabular}{|c|c|c|c|c|c|}
\hline Año & Votos & $\%$ & Año & Votos & $\%$ \\
\hline 1979 & 2.139 .603 & 12.8 & 1999 & 1.387 .900 & 6.5 \\
\hline 1983 & 1.500 .017 & 8.2 & 2003 & 1.394 .871 & 6.1 \\
\hline 1987 & 1.399 .364 & 7.2 & 2007 & 1.217 .030 & 5.5 \\
\hline 1991 & 1.579 .097 & 8.4 & 2011 & 1.424 .119 & 6.3 \\
\hline 1995 & 2.589 .780 & 11.7 & Media & $79-11$ & $8.1 \%$ \\
\hline
\end{tabular}

Fuente: Elaboración propia sobre datos MIR Para las EM 2011 www.elpais.com

Desde 1979 no se consiguió llegar a aquel techo electoral, que era $12.8 \%$, aunque en votos es 1995 la fecha del techo; un dato similar al de las E.G., cuyo techo en votos es 1996.

Es una media algo más alta que en las EG, a la baja. En 2003 IU mantuvo más de millón y medio de votos, contando los votos de Iniciativa per Catalunya, 2.601 concejales en toda España y 141 alcaldes. Procedían de 4 Comunidades fundamentalmente: de Andalucía 29\%, Cataluña 19\%, Madrid 15\% y Valencia 8\%. En 2007 volvió a bajar.

CUADRO 7: Concejales PCE/IU España/Andalucía

\begin{tabular}{|c|c|c|}
\cline { 2 - 3 } \multicolumn{1}{c|}{} & España & Andalucía \\
\hline 1979 & 3.753 & --- \\
\hline 1983 & 2.503 & --- \\
\hline 1987 & 2.315 & 1.089 \\
\hline 1991 & 2.614 & 1.033 \\
\hline 1995 & 3.493 & 1.329 \\
\hline 1999 & 2.295 & 1.129 \\
\hline 2003 & 2.601 & 1.089 \\
\hline 2007 & 2.595 & 1.090 \\
\hline 2011 & 2.248 & 1.104 \\
\hline
\end{tabular}

Fuente: MIR y www.historiaelectoral.com.

Entre 1979 y 2011, solo 9 ciudades con más de 75.000 habitantes han mantenido alcalde comunista. Son Córdoba (24 años), Sabadell (20), Santa Coloma de Gramanet (12), Algeciras, Coslada, Guadalajara, Teide, Badalona y Cornellá (4),

Se puede destacar al único alcalde de PCE/IU que lo es de su municipio desde 1979: Juan Manuel Sánchez Gordillo, alcalde de Marinaleda (Sevilla). José Antonio Barroso Toledo, alcalde de Puerto Real (Cádiz) lo fue desde el mismo año, aunque dejó de serlo en 2011. Este último no siempre ganó las elecciones con IU o PCE, sino también con candidaturas independientes o el PTE; están en el grupo de 68 alcaldes que lo son desde las primeras Elecciones municipales de 1979. Si destacamos aquéllos municipios cuyos alcaldes se han mantenido en la misma formación política -Alcaldes PCE/IU- en más de seis mandatos o legislaturas, tendríamos los siguientes datos:

9 mandatos: El Prat de Llobregat (Barcelona) y Marinaleda (Sevilla).

8 mandatos: Casares (Málaga) y Espera (Cádiz). 
7, Córdoba, La Rambla, Montalbán, Montemayor y Pedro Abad (Córdoba); La campana (Sevilla); Lupión (Jaén); Trebujena (Cádiz); Humilladero (Málaga); Grado (Asturias). Salinas (Alicante).

Hay además más de 600 municipios en Andalucía, Asturias, Cataluña, Castilla La Mancha, Castilla y León, Madrid, Comunidad Valenciana, Extremadura y Murcia, que han tenido, en otro número de legislaturas o en alguna, alcalde comunista.

\section{LOS SILOS DEL VOTO PCE/IU}

Por último, merece la pena tener en cuenta lo que sería el mapa electoral del voto comunista en España, vinculado a las Comunidades Autónomas. Se incluyen por eso a continuación los datos relativos a las circunscripciones con mayor apoyo PCE/IU, así como la media ponderada de apoyo popular en las 17 Comunidades Autónomas españolas.

CUADRO 8: Media ponderada Voto PCE/IU por Comunidades Autónomas 1977-2012

\begin{tabular}{|l|l|l|l|l|l|}
\cline { 2 - 6 } \multicolumn{1}{c|}{} & EG & EM & EA & EG y EM & EG-A-M \\
\hline España & $7.3 \%$ & $8.7 \%$ & - & $8 \%$ & \\
\hline Andalucía & $9.5 \%$ & $\mathbf{1 4 . 3 \%}$ & $11.9 \%$ & $11.6 \%$ & $11.8 \%$ \\
\hline Aragón & $6 \%$ & $5.8 \%$ & $6.2 \%$ & $5.9 \%$ & $6 \%$ \\
\hline Balurias & $\mathbf{1 1 . 6 \%}$ & $13.4 \%$ & $\mathbf{1 2 . 3 \%}$ & $\mathbf{1 2 . 5 \%}$ & $\mathbf{1 2 . 4 \%}$ \\
\hline Canarias & $4.8 \%$ & $3.8 \%$ & $2.8 \%$ & $4.3 \%$ & 3.8 \\
\hline Cantabria & $3.6 \%$ & $4 \%$ & $4 \%$ & $3.8 \%$ & $3.9 \%$ \\
\hline Castilla-La Mancha & $5.2 \%$ & $4.4 \%$ & $4.1 \%$ & $5.1 \%$ & $4.8 \%$ \\
\hline Castilla y León & $5.8 \%$ & $6.6 \%$ & $5 \%$ & $6.2 \%$ & $5.8 \%$ \\
\hline Cataluña & $4.7 \%$ & $4.8 \%$ & $4.9 \%$ & $4.8 \%$ & $4.7 \%$ \\
\hline Euzkadi & $4.1 \%$ & $10.4 \%$ & $8.3 \%$ & $9.2 \%$ & $8.9 \%$ \\
\hline Galicia & $2.8 \%$ & $4.4 \%$ & $4 \%$ & $4.6 \%$ & $4.4 \%$ \\
\hline Extremadura & $5.5 \%$ & $7.6 \%$ & $6.6 \%$ & $6.5 \%$ & $6.6 \%$ \\
\hline Madrid & $10 \%$ & $11 \%$ & $9.9 \%$ & $10.5 \%$ & $10.3 \%-$ \\
\hline Navarra & $5.1 \%$ & $4.1 \%$ & $5.2 \%$ & $4.6 \%$ & $4.8 \%$ \\
\hline Murcia & $6.2 \%$ & $7.6 \%$ & $4.5 \%$ & $6.9 \%$ & $6.1 \%$ \\
\hline Rioja & $4.1 \%$ & $3.2 \%$ & $3.7 \%$ & $3.6 \%$ & $3.7 \%$ \\
\hline Valencia & $7.3 \%$ & $8.1 \%$ & $7.6 \%$ & $7.7 \%$ & $7.6 \%$ \\
\hline
\end{tabular}

Fuente: Elaboración propia sobre datos MIR, CADPEA y El País para 2011 y 2012.

Resultados redondeados.

\subsection{Las provincias de mayor protagonismo $\mathrm{PCE} / \mathrm{IU}$}

Por último se incluye un cuadro de apoyos electorales a PCE/IU, en las cinco provincias entre ellas dos Comunidades Autónomas uniprovinciales- dónde es más significativo y coincide en las tres elecciones EG-EM-EA. Hay que añadir que, por Comunidades Autónomas son también estas las más fuertes en el voto PCE/IU. En función de esos datos se puede hablar de tres categorías de CCAA:

$1^{\text {a }}$ Las cinco citadas de mayor apoyo: Asturias, Cataluña (Barcelona), Andalucía (Córdoba), Madrid y Comunidad Valenciana (Valencia).

$2^{\mathrm{a}}$ Las de voto por debajo de la media, que puede considerarse el 8\% (Aragón, Cantabria, Castilla y León, Castilla La Mancha, Extremadura, Murcia y La Rioja).

$3^{\mathrm{a}}$ Aquéllas CCAA con predominio de candidaturas nacionalistas, en las que el voto PCE/IU es muy bajo o incluso inexistente (Navarra, P. Vasco, Baleares, Canarias y Galicia). 
CUADRO 9: Voto PCE/IU. Provincias significativas 1977-2012

\begin{tabular}{|c|c|c|c|c|c|}
\hline Votos/Prov. & Córdoba & Asturias & Barcelona & Madrid & Valencia \\
\hline Votos EG & 651.870 & 782.778 & 2.535 .082 & 3.211 .996 & 1.134 .258 \\
\hline$\%$ & $\mathbf{1 3 . 6 \%}$ & $11.2 \%$ & $9 \%$ & $10.1 \%$ & $8 \%$ \\
\hline Votos EM & 838.262 & 702.294 & 2.117 .866 & 2.281 .463 & 925.974 \\
\hline$\%$ & $\mathbf{2 4 . 6 \%}$ & $13.3 \%$ & $11.2 \%$ & $11 \%$ & $9.2 \%$ \\
\hline Votos EA & 665.657 & 565.965 & 2.158 .273 & 2.109 .910 & 1.298 .949 \\
\hline$\%$ & $\mathbf{1 7 . 8 \%}$ & $11.3 \%$ & $9.4 \%$ & $9.8 \%$ & $8.5 \%$ \\
\hline \% Medio & $\mathbf{1 8 . 7 \%}$ & $11.9 \%$ & $10.5 \%$ & $10.3 \%$ & $8.6 \%$ \\
\hline
\end{tabular}

Fuente. Elaboración propia sobre datos MIR, CADPEA, aunque no se han tenido en cuenta en esa relación, los datos municipales de 1979 y 1983, ni tampoco, según los datos de ese Centro de Estudios, los de las autonómicas de 1982; también en www.historiaelectoral.com, para Asturias y páginas Web de los Parlamentos de Andalucía www.parlamentodeandalucia.es, Madrid, www.asambleamadrid.es, Cataluña www.parlament.cat y Valencia www.cortsvalencianes.es

Salvo en las EG, en las que Asturias recibe más apoyos, aunque no en porcentaje, en las demás, sobre todo en las EM, es más consistente el apoyo PCE/IU en la provincia de Córdoba, más del $23 \%$.

El dato citado se completa con la referencia a las ciudades de España en que se mantiene un porcentaje de apoyo a las listas PCE/IU superior a la media estatal, que es del $7.5 \%$. Se incluyen en el cuadro las superiores al 12\%. Hay otras 14 que superan esa media. En la relación de ciudades aparecen, sobre todo, ciudades de las provincias más significativas, como puede comprobarse.

Se repite, por tanto, el protagonismo de los silos de voto PCE/IU en las provincias de Barcelona y Valencia y las Comunidades de Andalucía, Asturias y Madrid.

CUADRO 10: Media de apoyos electorales a PCE/IU en ciudades con más de 75.000 h y media superior a $12 \%$ en EG 1977-2011

\begin{tabular}{|l|l|l|l|}
\hline Rivas Vaciamadrid & 18.5 & Dos Hermanas & 13 \\
\hline Coslada & 16.8 & S. Coloma de Gramanet & 12.7 \\
\hline Córdoba & 15.5 & Avilés & 12.4 \\
\hline Getafe & 15.3 & Torrejón Ardoz & 12.3 \\
\hline Parla & 15.2 & S. Boi de Llobregat & 12.2 \\
\hline Cornellá de Llobregat & 14 & Alcalá de Henares & 12.1 \\
\hline Leganés & 13.5 & Gijón & 12 \\
\hline
\end{tabular}

Fuente: Elaboración propia sobre datos CADPEA y MIR redondeados

\section{LA ESPECIALIDAD DE LAS ELECCIONES EUROPEAS (EE)}

Parece oportuno aportar algunas referencias de las EE, que puedan confirmar o no, los datos que se han definido en torno al sustrato electoral comunista en España. Un espacio bastante claro de voto, aunque sometido a muchas turbulencias y cambios en su apoyo popular. Hay que recordar que en las EE los electores pueden votar a cualquier candidatura que se presente, puesto que solo existe ese único Colegio electoral, El Estado español.

En seis ocasiones se han celebrado Elecciones Europeas (EE) en España, desde que formamos parte de la Unión en 1986. Y los porcentajes de participación ya dan alguna pista de lo que significa nuestra integración europea: han ido disminuyendo esos porcentajes hasta el punto más bajo, que ha sido en 2009, del 55.1 de abstención; un solo dato que parece 
manifestar el carácter secundario de estas elecciones. No parece que exista demasiado interés por participar en una estructura supranacional, por mucho que condicione cada vez más ampliamente muchas decisiones claves para España.

Parece claro, por otro lado, que, aunque la circunscripción electoral es muy diferente del resto de lecciones, se repite el mismo comportamiento electoral, con escasas diferencias, que dan el mayor peso representativo al PSOE y al PP. De acuerdo con el cuadro $\mathrm{n}^{\circ} 12$ son los dos partidos más votados desde 1987, algo que, como ya se ha dicho, tiene mucho que ver con el sistema electoral D'Hondt, que define el procedimiento para trasformar votos en escaños.

CUADRO 11: Participación electoral E.Eur. 1987-2009

\begin{tabular}{|l|l|l|}
\hline & Participación & Abstención \\
\hline 1987 & $68.5 \%$ & $31.5 \%$ \\
\hline 1989 & $54.7 \%$ & 45.3 \\
\hline 1994 & $59.1 \%$ & 40.9 \\
\hline 1999 & $63.1 \%$ & 36.9 \\
\hline 2004 & $45.1 \%$ & 54.9 \\
\hline 2009 & $44.9 \%$ & 55.1 \\
\hline Media & $55.9 \%$ & $44.1 \%$ \\
\hline
\end{tabular}

Fuente: MIR y elaboración propia

CUADRO 12: Votos y \% a las cuatro candidaturas más votadas en las EE

\begin{tabular}{|c|c|c|c|c|c|c|c|}
\hline Año/Part. & IU \% & Votos & PSOE \% & Voto & PP \% & Votos & Otros \\
\hline 1987 & 5.3 & 1.011 .830 & 39.1 & 7.522 .706 & 24.7 & 4.747 .283 & CDS 10.3\% \\
\hline 1989 & 6.1 & 961.742 & 39.6 & 6.275 .552 & 21.4 & 3.395 .015 & CDS 7.2 \\
\hline 1994 & 13.4 & 2.497 .671 & 30.8 & 5.719 .707 & 40.1 & 7.453 .900 & CIU 4.7 \\
\hline 1999 & 5.8 & 1.221 .566 & 35.3 & 7.477 .823 & 39.7 & 8.410 .993 & CIU 4.4 \\
\hline 2004 & 4.2 & 643.136 & 43.5 & 6.741 .112 & 41.2 & 6.393 .192 & $* * \mathrm{G} 5.2$ \\
\hline 2009 & 3.7 & 588.248 & 38.8 & 6.141 .784 & 42.1 & 6.670 .377 & *C 5.1 \\
\hline
\end{tabular}

${ }^{*} \mathrm{C}$ : CEU: Candidatura Europea; proviene de la Europa de los Pueblos. ${ }^{* *} \mathrm{G}$ : GALEUSKA: Partidos nacionalistas moderados de Galicia y Euskadi. Como tercera fuerza política aparece IU, aunque hasta 1994 fue el CDS de Adolfo Suárez, partido ya desaparecido. Posteriormente CIU, Galeuska y La Candidatura Europea son las más votadas. Incluso en 2009 también consiguieron escaños UPyD y La misma CEU y Europa Verde. Fuente: MIR.

Por consiguiente, se trata de un sistema de partidos muy similar al que funciona en otras convocatorias electorales, a pesar de la enorme diferencia que existe en la circunscripción fijada. Eso da cuenta de la amplia fidelidad de los votantes PSOE, PP y PCE/IU.

En el número de escaños, si valoramos todas las elecciones, figura en primer lugar el PSOE (147 escaños), AP/PP (134), IU (24), CDS (12) Y CIU (11); además otras 11 o 12 candidaturas (en algún caso ha habido acuerdo entre dos coaliciones), han conseguido el resto de 24 escaños.

Por otro lado, en lo que se refiere al apoyo popular en las Europeas (EE) en España, es significativa su distribución por Comunidades Autónomas, en lo que se aprecia una práctica repetición de las consideraciones hechas anteriormente en relación con los silos del voto PCE/IU, de acuerdo con los datos que se exponen en el Cuadro 13.

Es significativo apreciar en este cuadro el techo electoral que alcanza IU, en todas las Comunidades, que es en las elecciones de 1994. En ellas casi se duplica el porcentaje de 
apoyo al PCE, con aumentos de entre 6 y 9 puntos; es más significativo en aquéllas Comunidades que no son silo del voto comunista, como País Vasco, Navarra y Cantabria.

CUADRO 13: Votos IU por CC.AA. en las EE \%.

\begin{tabular}{|l|c|c|c|c|c|c|c|}
\hline \multicolumn{1}{|c|}{ CCAA/Elec. } & E87\% & E89\% & E94\% & E99\% & E04\% & E09\% & $\begin{array}{c}\text { MEDI } \\
\text { A }\end{array}$ \\
\hline ANDalucia & 10.8 & 9.1 & $\mathbf{1 7 . 1}$ & 10.7 & 5.1 & 5.2 & $\mathbf{9 . 7}$ \\
\hline ARAgón & 3.4 & 6.3 & $\mathbf{1 3 . 7}$ & 4.0 & 3.1 & 3.5 & 5.7 \\
\hline ASTurias & 9.8 & 11.7 & $\mathbf{1 9 . 5}$ & 10.4 & 6.3 & 5.7 & $\mathbf{1 0 . 5}$ \\
\hline CANarias & 4.5 & 5.3 & $\mathbf{8 . 3}$ & 2.9 & 1.8 & 1.6 & 4.1 \\
\hline CanTabria & 3.4 & 4.2 & $\mathbf{1 2 . 3}$ & 5.0 & 2.5 & 2.0 & 4.9 \\
\hline CastillaLM & 4.6 & 5.4 & $\mathbf{1 0 . 5}$ & 4.1 & 2.7 & 3.0 & 5.1 \\
\hline C Y León & 2.5 & 4.0 & $\mathbf{1 1 . 0}$ & 5.0 & 2.4 & 2.3 & 4.5 \\
\hline CATaluña & 5.4 & 5.5 & $\mathbf{1 1 . 1}$ & 2.1 & 7.2 & 6.1 & 6.2 \\
\hline EXTremadura & 4.7 & 4.9 & $\mathbf{1 0 . 7}$ & 6.1 & 2.6 & 2.5 & 5.3 \\
\hline GALicia & 1.3 & 2.0 & $\mathbf{5 . 1}$ & 1.1 & 1.5 & 1.3 & 2.1 \\
\hline IBAleares & 2.2 & 3.5 & $\mathbf{9 . 9}$ & 3.8 & 2.4 & 2.6 & 4.1 \\
\hline LRIoja & 1.7 & 3.7 & $\mathbf{1 0 . 8}$ & 4.0 & 2.1 & 1.9 & 4.0 \\
\hline MADrid & 5.2 & 8.4 & $\mathbf{1 9 . 6}$ & 8.0 & 5.1 & 4.5 & $\mathbf{8 . 5}$ \\
\hline MURcia & 5.6 & 6.0 & $\mathbf{1 3 . 1}$ & 6.6 & 2.9 & 3.0 & 6.2 \\
\hline NAVarra & 1.4 & 3.1 & $\mathbf{1 2 . 8}$ & 5.4 & 4.3 & 3.3 & 5.1 \\
\hline PVAsco & 1.0 & 1.8 & $\mathbf{9 . 6}$ & 3.9 & 4.2 & 1.8 & 3.7 \\
\hline VALencia & 5.0 & 5.9 & $\mathbf{1 3 . 9}$ & 5.8 & 3.3 & 2.8 & 6.1 \\
\hline
\end{tabular}

En cambio en las medias por Comunidades siguen destacando las que son silo, esto es, Asturias, Andalucía y Madrid, por ese orden. Un dato que confirma lo que ya se expuso anteriormente en relación con el ámbito que es silo de voto comunista, que incluiría también Valencia y Cataluña, con menos apoyos en las EE.

\section{CONSIDERACIÓN FINAL}

Solo en las provincias de Barcelona, Córdoba, Madrid y Valencia, además de Asturias, se mantiene un peso significativo del voto PCE-IU. La tendencia, hasta 2012 era a la baja, aunque ha podido modificarse esta referencia en los tiempos de profunda crisis que vivimos.

El reto de IU sigue vigente, más allá de sus inercias y vicios organizativos, de sus pugnas continuas, de los imprescindibles cambios en las normas electorales, en la posibilidad de aglutinar, al menos parcialmente, el amplio descontento existente en la ciudadanía. Hay un espacio político significativo más allá del que ocupa el PSOE como fuerza hegemónica. Crecer es cambiar la dinámica electoral y tratar de encontrar las señas de identidad de la izquierda, que probablemente hoy ya no estén en los retos de la lucha de clases, sino en la búsqueda de alternativas al capitalismo, en la búsqueda de lo que une a la izquierda y no de lo que la separa, en la búsqueda de alternativas para el ser humano del siglo XXI.

\section{BIBLIOGRAFÍA}

AA.VV. (1981), Informe sociológico sobre el cambio político en España 1975-1981. IV Informe FOESSA. Volumen 1. Fundación FOESSA, Madrid, Ed. Suramérica. 
AA.VV. (2004), Organización territorial del Estado y Régimen electoral. CDP No 22-23. INAP, Madrid.

AA.VV. (2007), Historia del PCE. Madrid, FIM.

ALEJANDRE, J. (1990), "Consideraciones históricas sobre el derecho de sufragio en España". Anuario de la Facultad de Derecho, 10, Madrid, Universidad Complutense, pp. 287-300.

AJA, J. (2006), CIS. Estudios postelectorales, Elecciones generales 1982-1996. (CD-ROM) http://cis.sociol.es.

INFORME DE LA SITUACIÓN ELECTORAL DE LOS PARTIDOS DE LA IZQUIERDA EUROPEA. (2006), Ed. Digital, fim@yahoo.es

DIAZ DEL MORAL, J. (1929), "Historia de las agitaciones campesinas andaluzas. Córdoba. Antecedentes para una reforma agraria". Revista de derecho privado, Madrid.

MORENO GÓMEZ, F. (2008), El genocidio franquista en Córdoba. Barcelona, Crítica.

OÑATE, P. y OCAÑA, F. (1999), “Análisis de datos electorales”. Cuadernos Metodológicos del CIS, $\mathrm{n}^{\mathrm{o}} 27$, Madrid.

RAMIRO, L. (2004), Cambio y adaptación en la izquierda. La evolución del PCE y de IU (19862000).CIS. Monográficos, 207, Madrid.

RUIZ-HUERTA CARBONELL, A. (2009), Los ángulos ciegos. Una perspectiva crítica de la transición española. Madrid, Biblioteca Nueva y Fundación Ortega y Gasset.

SOTILLOS, I. (1997), El comportamiento electoral municipal español 1975-1995. CIS Monografias, 153, Madrid.

TRÍAS BEJARANO, J. (1978), "Los resultados de las elecciones legislativas del 15 de Junio de 1977 en la provincia de Córdoba. Un estudio por comarcas y municipios". Revista de Estudios Sociales, n ${ }^{\circ}$ 21-22, Madrid, pp. 157-208.

TUÑÓN DE LARA, M. (1971), La España del siglo XIX. París, Librería española.

\title{
Breve currículo:
}

\begin{abstract}
Alejandro Ruiz-Huerta Carbonell
Doctor en Derecho por la Universidad de Valladolid (1994). Licenciado en Ciencias Empresariales (Universidad de Deusto, 1973) Graduado en Derecho Constitucional y Ciencia Política (Centro de Estudios Constitucionales, 1978). Actividades profesionales: empresa privada (1973-1974); Colegio de Abogados de Madrid (1974-1979); Ministerio de Trabajo (1973); Congreso de los Diputados (1980-1983); Junta de Castilla y León (1983-1986). Universidades de Valladolid y Burgos, de la que fue primer Secretario General, y Profesor de Derecho Constitucional (1987-2008). Publicaciones: artículos y libros de la especialidad y de memoria personal: La memoria incómoda. Los abogados de atocha 1977-2002, en Dossoles, 2002; un estudio sobre la transición Los ángulos ciegos, en Biblioteca Nueva, 2009 y un estudio sobre El voto comunista en Córdoba, 1977-2012, Ediciones Universidad de Córdoba, 2013.
\end{abstract}

